\begin{tabular}{|c|l|}
\hline Title & Critical behavior of ac conductivity near the A nderson transition \\
\hline Author(s) & Shima, Hiroyuki; Nakayama, Tsuneyoshi \\
\hline Citation & $\begin{array}{l}\text { Physical Review B,60/20), 14066-14071 } \\
\text { https://doi.org/10.1103/PhysRevB.60.14066 }\end{array}$ \\
\hline Issue Date & 1999-11-15 \\
\hline Doc URL & http://hdl.handle.net/2115/6071 \\
\hline Rights & Copyright $\odot ~ 1999 A$ merican Physical Society \\
\hline Type & article \\
\hline File Information & PRB60_20.pdf \\
\hline
\end{tabular}

Instructions for use 


\title{
Critical behavior of ac conductivity near the Anderson transition
}

\author{
Hiroyuki Shima* and Tsuneyoshi Nakayama \\ Department of Applied Physics, Hokkaido University, Sapporo 060-8628, Japan
}

(Received 28 June 1999)

\begin{abstract}
We investigate the dynamic scaling behavior of ac conductivity $\sigma(\omega)$ in three-dimensional (3D) unitary and symplectic systems in addition to orthogonal one by means of large-scale simulations. It is demonstrated that the ac conductivity near the Anderson transition behave as $\sigma(\omega) \propto \omega^{\delta}(\delta \simeq 1 / 3)$ for all of the above systems. Numerical calculations are performed by an efficient algorithm based on the forced oscillator method (FOM), which enables us to accurately treat large-scale quantum systems with less computational effort. The values of the exponents $\delta$ are determined by the finite-time scaling method for the FOM. [S0163-1829(99)11943-X]
\end{abstract}

\section{INTRODUCTION}

The scaling arguments of localization ${ }^{1,2}$ have stimulated many works on both static and dynamic behavior of disordered electron systems, especially on the Anderson transition. The existence of this transition essentially depends only on the dimensionality and the symmetries of the systems. Three-dimensional (3D) systems generally show the Anderson transition as a function of the strength of disorder and the Fermi energy, and their critical behavior are classified into three universality classes according to the basic symmetry of the Hamiltonian. The systems being invariant under spin rotation in addition to time-reversal symmetry constitute the orthogonal class, while the systems being invariant under time reversal but having no spin-rotation symmetry belong to the symplectic class. The rest forms the unitary class characterized by the absence of time-reversal symmetry. ${ }^{3}$

Many numerical works have contributed to reveal both the static and dynamic behavior of the transition through the investigations of localization length, ${ }^{4,5}$ diffusion of wave packets, ${ }^{6-9}$ and level statistics. ${ }^{10-12}$ Linear response functions also provide insight into the dynamic properties of this quantum phase transition. Calculations of linear response functions for quantum systems normally require the evaluations of all eigenvalues and corresponding eigenvectors. The direct diagonalization techniques to calculate these quantities remain limited to systems of modest size because their computing time and memory space grows quite rapidly as the system size $L$ become large. Especially, for 3D cases, the conventional routine is not suitable for computing linear response functions of large-scale quantum systems. For example, the ac conductivity $\sigma(\omega)$ in 3D systems near the transition was suggested to obey the power law $\sigma(\omega) \propto \omega^{1 / 3}$ by Wegner, ${ }^{13}$ while this behavior for orthogonal system was not numerically verified until the work by Lambrianides and Shore. ${ }^{14}$ They have evaluated the Kubo-Greenwood formula ${ }^{15,16}$ by directly calculating eigenvectors of the system with the diagnalization method, so the system sizes $N$ $\left(=L^{3}\right)$ treated were very limited $(L=6-14)$, indicating the relevance of the finite-size effect.

Recently, we have developed a method to calculate linear response functions for quantum systems described by largescale Hamiltonian matrices. ${ }^{17}$ This algorithm is based on the forced oscillator method (FOM), ${ }^{18,19}$ which enables us to ef- ficiently compute the spectral density of states, eigenvalues, and their eigenvectors of the systems described by very large matrices. ${ }^{20}$ We have further developed a new scaling approach, utilizing the characteristics of the FOM, to determine the dynamical exponent of the ac conductivity $\sigma(\omega) \propto \omega^{\delta}$ near the Anderson transition with high speed and accuracy. ${ }^{17,21}$

In this paper, we calculate the dynamical exponents in 3D unitary and symplectic systems. For these systems different from orthogonal one, the Hamiltonian matrices become complex and/or posses spinor components so that it is not easy to calculate $\sigma(\omega)$ with conventional methods. Within our knowledge, the present work is the first numerical realization of the $\omega^{1 / 3}$ behavior of $\sigma(\omega)$ in unitary and symplectic systems.

The outline of this paper is as follows. In Sec. II, we explain the model Hamiltonian and an efficient algorithm to compute linear response functions. Section III presents the finite-time scaling method for the dynamical exponent of $\sigma(\omega) \propto \omega^{\delta}$, and shows the calculated results for the $3 \mathrm{D}$ Anderson model near the transition. Section IV describes the scaling theory for the ac conductivity $\sigma(\omega)$ that gives the $\omega^{1 / 3}$ dependence close to the transition. We also discuss about the $\omega$ dependence of $\sigma(\omega)$ in the very lower frequency region relevant to the anomalous diffusion exponent $\eta$. A summary is given in this section.

\section{METHOD}

\section{A. Model}

We consider noninteracting electron systems on a simple cubic lattice with disordered potentials. The Hamiltonian of the system is given by

$$
H=\sum_{i, \sigma} W_{i, \sigma}|i \sigma\rangle\left\langle i \sigma\left|+\sum_{i, \sigma ; j, \sigma^{\prime}} V_{i, \sigma ; j, \sigma^{\prime}}\right| i \sigma\right\rangle\left\langle j \sigma^{\prime}\right|,
$$

where $i$ denotes the lattice site, and $\sigma$ the spin, respectively. We set the lattice constant to be unity and only the nearestneighbor coupling is taken into account. The on-site potentials $\left\{W_{i}\right\}$ are assumed to be distributed independently, and the distribution is taken to be uniform in the range $[-W / 2, W / 2]$. In the orthogonal case, $V_{i, \sigma ; j, \sigma^{\prime}}=V \delta_{\sigma, \sigma^{\prime}}$ is real, while $V_{i, \sigma ; j, \sigma^{\prime}}$ is $V \exp \left(i \phi_{i, j}\right) \delta_{\sigma, \sigma^{\prime}}$ in the unitary case 
with the Peierls phase factor $\phi_{i, j}$. In both cases, no spin flip process is included. In the symplectic case, the hopping energy is described by

$$
V_{i, \sigma ; i-k, \sigma^{\prime}}=V\left[\exp \left(-i \theta \sigma_{k}\right)\right]_{\sigma, \sigma^{\prime}}, \quad k \equiv \hat{x}, \hat{y}, \hat{z}
$$

where $\sigma_{k}$ are Pauli matrices. ${ }^{22}$ We set the hopping amplitude $V$ the energy unit.

\section{B. ac conductivity and resonance function}

Direct diagonalization for 3D systems requires huge computing time, especially when the matrix elements of the Hamiltonian have several degrees of freedom due to applied magnetic fields or spin-orbit interaction. Without carrying out the direct diagonalization, we employ an efficient method based on the FOM to calculate the ac conductivity $\sigma(\omega) .{ }^{17}$ The characteristics of the method are its simplicity, speed, memory efficiency, and wide applicability to general quantum systems. This method is based on solving the Schrödinger equation numerically under a periodic external force, so that it can be easily vectorized and parallelized for implementation in an array-processing modern supercomputer.

Let us consider the system described by Eq. (1), whose abbreviated form is given by

$$
H=\sum_{i j} H_{i j}|i\rangle\langle j|,
$$

and an electron state $|\Psi(t)\rangle$ is expanded as

$$
|\Psi(t)\rangle=\sum_{i} a_{i}(t)|i\rangle
$$

where the index $i$ represents both the site and the spin for simplicity.

For calculating the ac conductivity $\sigma(\omega)$, we impose a perturbation $H^{\prime}$ to the system expressed by

$$
H^{\prime}=-\frac{J}{2}\left(A_{0} e^{-i \omega t}+\text { c.c. }\right),
$$

where $J$ is the current operator and $A_{0}$ is the amplitude of the external vector potential, respectively. c.c. indicates a complex conjugate.

Substituting Eqs. (3) and (5) into the Schrödinger equation for $|\Psi(t)\rangle$ and applying the time-dependent first-order perturbation theory by putting $a_{i}(t)=a_{i}^{(0)}(t)+a_{i}^{(1)}(t)$, one has the linear differential equation with periodic external force,

$$
\begin{aligned}
i \hbar & \frac{d a_{i}^{(1)}(t)}{d t}-\sum_{j} H_{i j} a_{j}^{(1)}(t) \\
& =-\frac{\hbar}{2}\left(F_{i} e^{-i \omega t}+\widetilde{F}_{i} e^{i \omega t}\right) e^{-i \omega_{\lambda 0} t},
\end{aligned}
$$

where the definitions are

$$
F_{i} \equiv \sum_{j} \frac{A_{0}}{\hbar} J_{i j} \varphi_{j}\left(\omega_{\lambda 0}\right), \quad \widetilde{F}_{i} \equiv \sum_{j} \frac{A_{0}^{*}}{\hbar} J_{i j} \varphi_{j}\left(\omega_{\lambda 0}\right),
$$

and $\varphi_{j}\left(\omega_{\lambda 0}\right) \equiv\left\langle j \mid \omega_{\lambda 0}\right\rangle$, where $\left|\omega_{\lambda 0}\right\rangle$ is the initial eigenvector belonging to the eigenvalue $\hbar \omega_{\lambda 0}$ of the matrix $\left\{H_{i j}\right\}$. We have used in Eq. (6) the definition given by $a_{j}^{(0)}(t)$ $=\varphi_{j}\left(\omega_{\lambda 0}\right) e^{-i \omega_{\lambda 0} t}$.

We introduce the resonance function defined by the sum of the squared amplitude;

$$
E\left(\omega, \omega_{\lambda 0}, t\right) \equiv \sum_{i}\left|a_{i}^{(1)}(t)\right|^{2} .
$$

Substituting the solution of Eq. (6) under the initial condition $a_{i}^{(1)}(0)=0$ into Eq. (8), one has

$$
\begin{aligned}
E\left(\omega, \omega_{\lambda 0}, t\right)= & \sum_{\lambda}\left|\sum_{i} F_{i} \varphi_{i}^{*}\left(\omega_{\lambda}\right)\right|^{2} \\
& \times \frac{\sin ^{2}\left\{\left(\omega_{\lambda}-\omega_{\lambda 0}-\omega\right) t / 2\right\}}{\left(\omega_{\lambda}-\omega_{\lambda 0}-\omega\right)^{2}},
\end{aligned}
$$

where the contribution from the second term on the right hand-side of Eq. (6) is ignored, since we consider the case of zero temperature (See Ref. 17).

The eigenvectors contributing to the sum on $\lambda$ in Eq. (9) are those whose frequencies lie within about $\pm(2 \pi / t)$ of $\omega_{\lambda 0}+\omega$, where $t$ is the time interval. Taking a sufficient large time interval $t$, only eigenmodes in a narrow band of frequencies on the scale of $\omega_{\lambda 0}+\omega$ contribute to the sum in Eq. (9). Thus, we have

$E\left(\omega, \omega_{\lambda 0}, t\right)=\frac{\pi t\left|A_{0}\right|^{2}}{2 \hbar^{2}} \sum_{\lambda}\left|\left\langle\omega_{\lambda}|J| \omega_{\lambda 0}\right\rangle\right|^{2} \delta\left(\omega_{\lambda}-\omega_{\lambda 0}-\omega\right)$,

where the following relation is used

$$
\sum_{i} F_{i} \varphi_{i}^{*}\left(\omega_{\lambda}\right)=\frac{A_{0}}{\hbar}\left\langle\omega_{\lambda}|J| \omega_{\lambda 0}\right\rangle
$$

The resonance function expressed by Eq. (10) can be related to the ac conductivity $\sigma(\omega)$ as follows. The explicit form of $\sigma(\omega)$ is

$$
\begin{aligned}
\sigma(\omega)= & \frac{2 \pi}{\hbar \omega L^{d}} \sum_{\lambda 0} \sum_{\lambda}\left|\left\langle\omega_{\lambda}|J| \omega_{\lambda 0}\right\rangle\right|^{2}\left[f\left(\omega_{\lambda 0}\right)-f\left(\omega_{\lambda}\right)\right] \\
& \times \delta\left(\omega_{\lambda}-\omega_{\lambda 0}-\omega\right)
\end{aligned}
$$

where $f\left(\omega_{\lambda}\right)$ is the Fermi distribution function that becomes a step function at zero temperature. The spin freedom is taken into account. Comparing Eqs. (10) and (12), one has the key equation relating the resonance function and the ac conductivity as,

$$
\sigma(\omega)=\frac{4 \hbar}{\omega t L^{d}} \sum_{\omega_{\lambda 0}=\omega_{F}-\omega}^{\omega_{F}} E\left(\omega_{\lambda 0}, \omega, t\right)
$$

where we have set $\left|A_{0}\right|=1$ without loss of generality and the definition of the Fermi frequency is $\omega_{F}=E_{F} / \hbar$. 


\section{Time development}

We use the modified Euler method to obtain the time development of the first-order linear differential Eq. (6). This method makes calculations very efficient and accurate. However, we cannot apply this method in the same way as used in Ref. 17 where the matrix elements $\left\{H_{i j}\right\}$ and the external forces $\left\{F_{i}\right\}$ are real numbers due to the orthogonal symmetry, while these are complex for unitary and symplectic systems. Here, we divide the function $a_{i}^{(1)}(t)$ in Eq. (6) into the real part $x_{i}(t)$ and imaginary one $y_{i}(t)$, and map each of the first-order differential equations to the second-order one as

$$
\begin{gathered}
\frac{d^{2} x_{i}(T)}{d T^{2}}+\sum_{j} D_{i j}^{R} y_{j}(T)+\sum_{j} D_{i j}^{I} x_{j}(T) \\
=\frac{1}{2}\left\{F_{i}^{R} \sin (\Omega T)-F_{i}^{I} \cos (\Omega T)\right\},
\end{gathered}
$$

and

$$
\begin{gathered}
\frac{d^{2} y_{i}(T)}{d T^{2}}-\sum_{j} D_{i j}^{R} x_{j}(T)+\sum_{j} D_{i j}^{I} y_{j}(T) \\
=\frac{1}{2}\left\{F_{i}^{R} \cos (\Omega T)+F_{i}^{I} \sin (\Omega T)\right\} .
\end{gathered}
$$

We have defined $\Omega=\sqrt{\omega_{\lambda 0}+\omega+\omega_{0}}, T=2 \Omega t$, and $D_{i j}$ $=\omega_{0} \delta_{i j}+H_{i j} / \hbar$, respectively. $D_{i j}^{R}$ and $D_{i j}^{I}$ represent a real and imaginary part of $D_{i j}$. By adding the positive quantity $\hbar \omega_{0} \geqslant\left|\hbar \omega_{\lambda \text { min }}\right|$, the minimum eigenvalues of $D_{i j}$ become always positive (See Ref. 20 for details). The reason of the mapping to the second-order differential equations from the first-order one is that we use the modified Euler method in order to obtain the time development of Eqs. (14) and (15) as follows.

We define $\mathrm{\vee}_{i}(T)=d x_{i}(T) / d T$ and discretize a time $T$ with a step $\tau$, we have

$$
\begin{gathered}
x_{i}(n+1)=x_{i}(n)+\mathrm{v}_{i}(n+1) \tau, \\
\mathrm{v}_{i}(n+1)=\mathrm{v}_{i}(n)-\sum_{j} D_{i j}^{I} x_{j}(n)-\sum_{j} D_{i j}^{R} y_{j}(n) \\
+\frac{1}{2}\left\{F_{i}^{R} \sin (\Omega n \tau)-F_{i}^{I} \cos (\Omega n \tau)\right\},
\end{gathered}
$$

where the integer $n$ represents the number of time step. The time development of the imaginary part $y_{i}(t)$ can be obtained as well. It should be noted that the second term on the right-hand side of Eq. (16) depends on the number $n+1$ of time steps, namely, defined by the retarded finite-difference form, which is different from the standard Euler method defined by the advanced one as used for Eq. (17). This choice in Eq. (16) makes calculations, by taking the time step $\tau$ satisfying the condition $\omega_{\lambda \text { max }} \tau<2$, very efficient and accurate, as discovered by Williams and Maris. ${ }^{18}$

We have described in this section the method of computing the ac conductivity for systems described by large-scale Hamiltonian matrices. We emphasize that this algorithm enables us to directly calculate conductivity without making the temporal Fourier transform of the response function or calculating all of the intermediate states $\varphi_{i}\left(\omega_{\lambda}\right)$ relevant to the formula (12).

In actual calculations, we have prepared the limited numbers of the initial eigenmodes $\varphi_{i}\left(\omega_{\lambda_{0}}\right)$ within the range $\omega_{F}$ $-\omega \leqslant \omega_{\lambda 0} \leqslant \omega_{F}$ by original FOM, which enables us to calculate quite accurately the selected eigenmode belonging to an arbitrary eigenfrequency. ${ }^{19}$

\section{FINITE-TIME SCALING APPROACH}

\section{A. Scaling form of the resonance function}

In this section, we present the finite-time scaling approach to determine the critical exponent of the ac conductivity $\sigma(\omega) \propto \omega^{\delta}$ and the calculated results of $\delta$ for unitary and symplectic systems. This scaling approach is based on the fact that the number of eigennmodes contributing to the sum on $\lambda$ in Eq. (9) depends on the resonance width of sinclike function $4 \pi / t$, which is inversely proportional to the time interval $t$ for which the external force is applied.

The explicit form of the resonance function is given by Eq. (9) as

$$
\begin{aligned}
E\left(\omega_{\lambda 0}, \omega, t\right)= & \frac{1}{\hbar^{2}} \int d\left(\hbar \omega_{\lambda}\right) \rho\left(\omega_{\lambda}\right)\left|\left\langle\omega_{\lambda}|J| \omega_{\lambda 0}\right\rangle\right|^{2} \\
& \times \frac{\sin ^{2}\left\{\left(\omega_{\lambda}-\omega_{\lambda 0}-\omega\right) t / 2\right\}}{\left(\omega_{\lambda}-\omega_{\lambda 0}-\omega\right)^{2}},
\end{aligned}
$$

where the density of states $\rho\left(\omega_{\lambda}\right)$ is introduced by the definition $\Sigma_{\lambda}=L^{d} \int d\left(\hbar \omega_{\lambda}\right) \rho\left(\omega_{\lambda}\right)$. We assume that the ac conductivity obeys the power law close to the transition as $\sigma(\omega) \propto \omega^{\delta}$. Under this condition, Eq. (18) should be, for a sufficiently large time interval $t$,

$$
\frac{E\left(\omega_{\lambda 0}, \omega, t\right)}{t} \propto \int d \omega_{\lambda}\left(\omega_{\lambda}-\omega_{\lambda 0}\right)^{\delta} \delta\left(\omega_{\lambda}-\omega_{\lambda 0}-\omega\right) \propto \omega^{\delta} .
$$

We have used the fact that $\rho\left(\omega_{\lambda}\right)$ is nearly constant in the band center. For the short time interval $t$, the resonance width in Eq. (18) become wider than the bandwidth and one yields

$$
\frac{E\left(\omega_{\lambda 0}, \omega, t\right)}{t} \propto t^{-\delta}
$$

From these two extreme cases, the scaling form of the resonance function, or equivalently, of the ac conductivity becomes,

$$
\sigma(\omega, t) \propto t^{-\delta} G(\omega t)
$$

where the asymptotic form of $G(z)$ should be

$$
G(z)=\left\{\begin{array}{cc}
z^{\delta}, & z \gg 1 \\
\text { const, } & 0<z \ll 1 .
\end{array}\right.
$$

We have demonstrated the efficiency of the finite-time scaling approach to determine the critical exponent of the ac conductivity $\sigma(\omega) \propto \omega^{\delta}$ at the Anderson transition for an orthogonal system with high accuracy. ${ }^{21}$ 


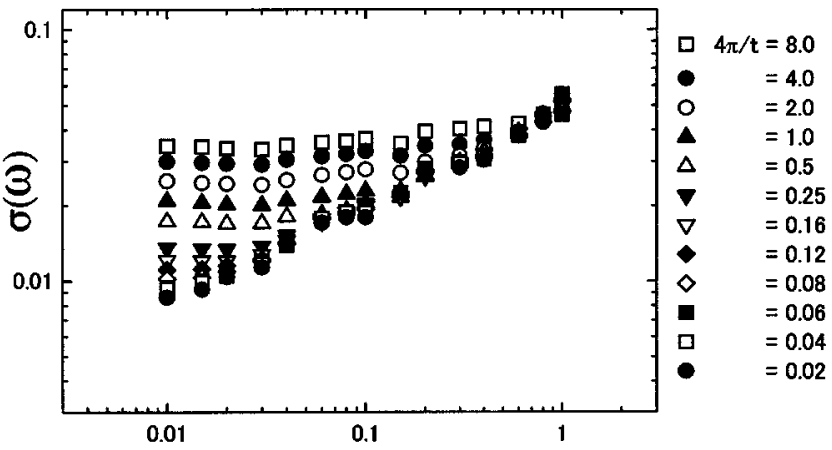

(a)

$\omega$

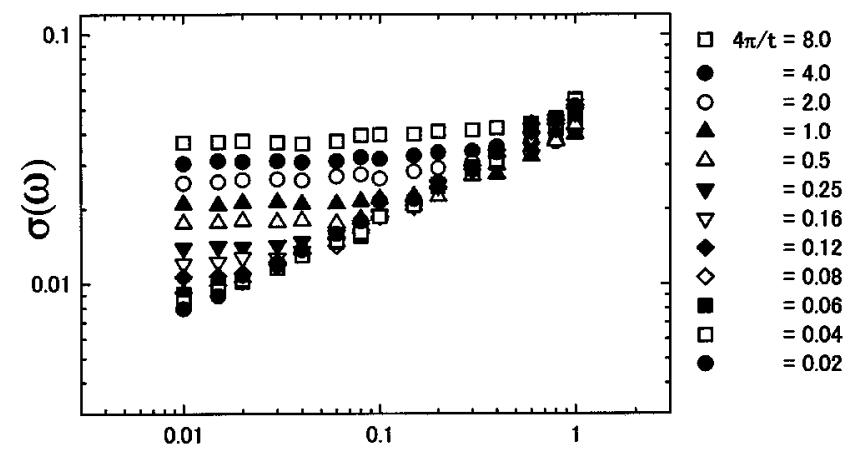

(b)

w

FIG. 1. Calculated results of $\sigma(\omega)$ for the 3D (a) unitary and (b) symplectic systems for various time interval $t$. The system size is taken as $L=30$.

\section{B. Results for unitary and symplectic systems}

In order to discuss the dynamic properties in the vicinity of the Anderson transition, we set the disorder strength $W$ $=W_{C}=17.9$ (Ref. 23) for the unitary case, assuming uniform magnetic fields to be parallel to the $z$ direction, and a magnetic flux penetrating through a $x-y$ plane unit cell is set to be 0.2 times the flux quantum. For the symplectic case, we set $\theta=\pi / 6$ in Eq. (2), and $W$ is set to the critical value $W_{C}$ $=19.0{ }^{12}$ The Fermi energy $E_{F}$ is fixed to the band center. Actual simulations have been performed for systems with $30 \times 30 \times 30$ lattice sites for both cases. In each case, averaging over 20 independent realization of random potentials has been performed.

Figure 1 presents the calculated results of $\sigma(\omega)$ for both cases taking various time interval $t=\pi / 2 \sim 200 \pi$. The corresponding resonance widths become $4 \pi / t=0.02 \sim 8.0$ in units of $V=1$. We see from Fig. 1 that the calculated results follow the $\omega^{1 / 3}$ behavior with increasing time interval $t$ over two orders of magnitude on frequency. Figure 2 shows the scaling function $G(z)$ defined in Eq. (20). The most likely fit is determined by $\chi^{2}$ statistic, and the confident intervals for fitting parameters were estimated from the bootstrap procedure. The calculated results of the exponent is $\delta=0.34$ \pm 0.02 for unitary case and $\delta=0.34 \pm 0.01$ for symplectic case, respectively. These values agree well with the prediction of the scaling theory for the ac conductivity $\sigma(\omega) .^{13}$

\section{SUMMARY AND DISCUSSION}

The scaling hypothesis for the ac conductivity $\sigma(\omega)$ for the finite system size $L$ is expressed as ${ }^{24}$
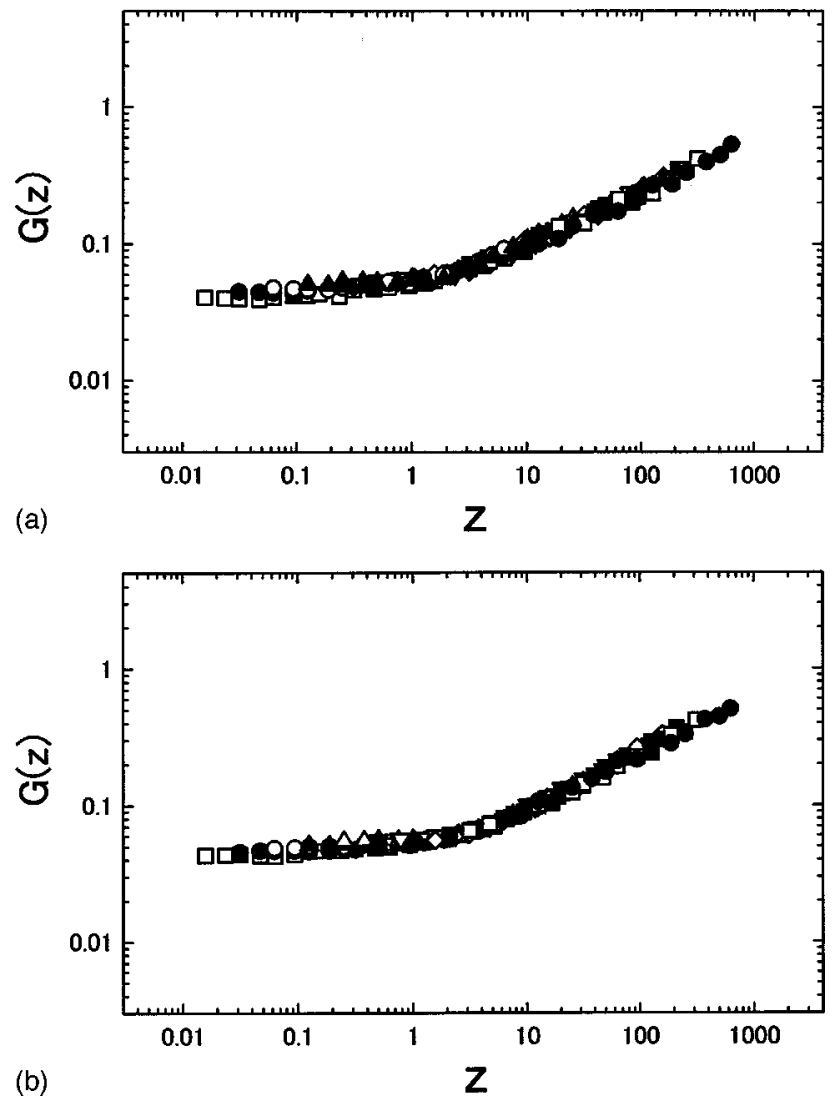

FIG. 2. The scaling function $G(z)$ introduced in Eq. (20) for (a) unitary and (b) symplectic systems. The estimated values of dynamical exponents are $\delta=0.34 \pm 0.02$ for unitary case, and $\delta$ $=0.34 \pm 0.01$ for symplectic case.

$$
\sigma(\omega)=\frac{e^{2}}{\hbar} L^{2-d} f\left(\frac{L}{\xi}, \frac{L_{\omega}}{\xi}\right) .
$$

This hypothesis is based on the fact that there are three length scales characterizing the system, namely, the correlation length $\xi$, the system size $L$, and a characteristic length $L_{\omega} . L_{\omega}$ means the distance an electron diffuses in the system during one cycle of an applied field,

$$
L_{\omega}=\sqrt{\frac{D(\omega)}{\omega}},
$$

where $D(\omega)$ is the frequency-dependent diffusion coefficient. ${ }^{25}$ When the system is very close to the transition point and $L_{\omega} \ll \xi, L_{\omega}$ becomes the only relevant length and $\sigma(\omega)$ scales like

$$
\sigma(\omega) \propto L_{\omega}^{2-d}=\left\{\frac{\omega}{D(\omega)}\right\}^{(d-2) / 2},
$$

where the thermodynamic limit $(L \rightarrow \infty)$ is taken. Noting that $\sigma(\omega)$ and $D(\omega)$ are related via the Einstein relation $\sigma(\omega)$ $=e^{2} \rho\left(E_{F}\right) D(\omega)$, one obtains

$$
\sigma(\omega) \propto \omega^{(d-2) / d} .
$$

This form has been predicted by Wegner. ${ }^{13}$ For $d=3$, Eq. (25) is reduced to the power law $\sigma(\omega) \propto \omega^{1 / 3}$, which agrees with our results. 
One can give other interpretation for $L_{\omega}$ as

$$
L_{\omega}=\left\{\rho\left(E_{F}\right) \hbar \omega\right\}^{-1 / d}=L\left(\frac{\hbar \omega}{\Delta}\right)^{-1 / d},
$$

where $\Delta$ is the mean level spacing of the system. $L_{\omega}$ is thus equivalent to the size of a fictitious system with the mean level spacing $\hbar \omega$. Strictly speaking, length scales defined by Eqs. (23) and (26) are different only by a factor $g^{* 1 / d}$, where $g^{*}$ is the dimensionless conductance at the transition. ${ }^{26}$ Provided that the length $L_{\omega}$ is only relevant, namely, shorter than $L$, the energy $\hbar \omega$ should be larger than the mean level spacing $\Delta$.

Let us consider the wave-number-dependent ac conductivity $\sigma(q, \omega)$ in order to discuss the behavior in the lower frequency region. In the lower frequency region, where $\hbar \omega$ is smaller than $\Delta, \sigma(\omega)$ is expected to show a different behavior related to the anomalous diffusion exponent $\eta$. The general conductivity $\sigma(q, \omega)$ is given by

$$
\sigma(q, \omega) \propto \frac{D(q, \omega)}{1+\left\{q^{2} D(q, \omega) / \omega\right\}^{2}},
$$

where $D(q, \omega)$ is the generalized diffusion coefficient. ${ }^{25}$ The scaling form of $D(q, \omega)$ near the Anderson transition presented by Chalker et al. $^{27}$ is expressed as

$$
D(q, \omega) \propto q^{d-2} f(x) \quad: \quad x \equiv\left(q L_{\omega}\right)^{d},
$$

where the system size $L$ and the correlation length $\xi$ are assumed to be larger than $L_{\omega}$ and $1 / q$. The asymptotic behavior of the function $f(x)$ is

$$
f(x) \propto \begin{cases}x^{(2-d) / d} ; & x \ll 1 \\ x^{-\eta / d} ; & x \gg 1 .\end{cases}
$$

The exponent $\eta$ implies the strong fluctuation of the amplitudes of wave functions near the transition.

For $q L_{\omega} \ll 1$ where $D(q, \omega)$ depends only $\omega$, the conductivity behaves in the same way as in Eq. (25). While for $q L_{\omega} \gg 1$ where the wave length $\sim 1 / q$ is smaller than $L_{\omega}$, the conductivity shows an anomalous behavior as

$$
\sigma(q, \omega) \propto q^{d-2}\left(\frac{\omega}{q^{d}}\right)^{2-\eta / d} .
$$

In finite size systems, the size $L$ provides the lower cutoff for the wave number $q$. Thus, the $q$ dependence of $\sigma(q, \omega)$ can be obtained from an analysis of the $L$ dependence of the ac conductivity. For $d=3$, one expects that the conductivity shows $\sigma(\omega, L) \propto 1 / L \cdot\left(\omega L^{3}\right)^{2-\eta / 3}$ for $\omega L^{3}<1$, which means that the frequency $\omega$ is smaller than the mean level spacing
$\Delta$. In the present work, we have calculated the ac conductivity $\sigma(\omega)$ for the energy range $16 \Delta \leqslant \hbar \omega \leqslant 1600 \Delta$ since $\Delta$ is about $6.4 \times 10^{-4}$ in the present system $(L=30)$. In addition, it should be noted that the resonance widths $4 \pi / t$ have been set more twenty times larger than $\Delta$ to remove finite size effects. In order to gain insight into the value of $\eta$, one should set $\hbar \omega$ and $4 \pi / t$ comparable to $\Delta$ because the discreteness of energy level due to the finite size effect becomes relevant. In such a condition the direct diagonalization is suitable to calculate $\sigma(\omega, L)$ for small systems, while it is evident that the algorithm we used in this paper is powerful for calculating $\sigma(\omega, L)$ for large-scale systems.

We mention about the numerical work by Brandes et al. ${ }^{26}$ on the anomalous diffusion exponent $\eta$ in $3 \mathrm{D}$ orthogonal system. They have calculated the function defined by

$$
Z\left(E, E^{\prime}\right)=\int d^{d} x\left|\Psi_{E}(x)\right|^{2}\left|\Psi_{E^{\prime}}(x)\right|^{2}
$$

for eigenstates with energy $E$ and $E^{\prime}$ within the region $\Delta$ $\leqslant \hbar \omega \equiv\left|E-E^{\prime}\right| \leqslant 300 \Delta$, and demonstrated the power law $Z(\omega) \propto \omega^{-\eta / d}$. They have used the Lanczos method to directly diagonalize the system $L=40$ and the estimated value $\eta \sim 1.5$ is close to that of other works on the fractal dimension of the wave function $D_{2}(=d-\eta){ }^{8,28,29}$ According to their results, the crossover frequency $\omega_{C}$ separating the two regions expressed by Eqs. (25) and (30) is equal to $300 \Delta$ or larger, namely, $\omega_{C} \sim 0.2$ for the system size $L=30$. Thus, $\sigma(\omega)$ is expected to behave like $\propto \omega^{1.5}$ in the region $\omega$ $\leqslant 0.2$ for $L=30$, but we could not observe such a behavior in our calculated results. There has been no work on the quantitative determination of the frequency $\omega_{C}$ in $3 \mathrm{D}$ system so it will be presented elsewhere in near future.

To summarize, we have calculated $\sigma(\omega)$ near the Anderson transition in 3D unitary and symplectic systems and the dynamical exponent $\sigma(\omega) \propto \omega^{\delta}$ has been determined by the finite-time scaling approach based on the FOM. The estimated values clearly take $\delta \simeq 1 / 3$ for both systems as well as the case for orthogonal one, which agree with the prediction from the scaling argument.

\section{ACKNOWLEDGMENTS}

We would like to thank Kousuke Yakubo for fruitful discussion. One of the authors (H.S.) was supported in part by the Japan Society for the Promotion of Science for Young Scientists. This work was supported in part by a Grant-inAid for Scientific Research from the Japan Ministry of Education, Science and Culture. Numerical calculations were partially performed on the FACOM VPP 500 of the Supercomputer Center, Institute of Solid State Physics, University of Tokyo.

\footnotetext{
*Electronic address: shima@eng.hokudai.ac.jp

${ }^{1}$ P.W. Anderson, Phys. Rev. 109, 1492 (1958).

${ }^{2}$ E. Abrahams, P.W. Anderson, D.C. Licciardello, and T.V. Ramakrishnan, Phys. Rev. Lett. 42, 673 (1979).

${ }^{3}$ F.J. Dyson, J. Math. Phys. 3, 140 (1962); 3, 157 (1962); 3, 166 (1962).

${ }^{4}$ A. MacKinnon and B. Kramer, Z. Phys. B 53, 1 (1983).

${ }^{5}$ K. Slevin and T. Ohtsuki, Phys. Rev. Lett. 78, 4083 (1997).
}

${ }^{6}$ B. Kramer and A. MacKinnon, Rep. Prog. Phys. 56, 1469 (1993).

${ }^{7}$ T. Kawarabayashi and T. Ohtsuki, Phys. Rev. B 51, 10897 (1995); 53, 6975 (1996).

${ }^{8}$ T. Ohtsuki and T. Kawarabayashi, J. Phys. Soc. Jpn. 66, 314 (1997).

${ }^{9}$ B. Huckestein and R. Klesse, Phys. Rev. B 59, 9714 (1999).

${ }^{10}$ B.I. Shklovskii, B. Shapiro, B.R. Sears, P. Lambrianides, and H.B. Shore, Phys. Rev. B 47, 11487 (1993). 
${ }^{11}$ M. Batsch, L. Schweitzer, I.Kh. Zharekeshev, and B. Kramer, Phys. Rev. Lett. 77, 1552 (1996).

${ }^{12}$ T. Kawarabayashi, T. Ohtsuki, K. Slevin, and Y. Ono, Phys. Rev. Lett. 77, 3593 (1996).

${ }^{13}$ F.J. Wegner, Z. Phys. B 25, 327 (1976).

${ }^{14}$ P. Lambrianides and H.B. Shore, Phys. Rev. B 50, 7268 (1994).

${ }^{15}$ R. Kubo, J. Phys. Soc. Jpn. 12, 570 (1957).

${ }^{16}$ A.D. Greenwood, Proc. Phys. Soc. London 71, 585 (1958).

${ }^{17}$ T. Nakayama and H. Shima, Phys. Rev. E 58, 3984 (1998).

${ }^{18}$ M.L. Williams and H.J. Maris, Phys. Rev. B 31, 4508 (1985).

${ }^{19}$ K. Yakubo, T. Nakayama, and H.J. Maris, J. Phys. Soc. Jpn. 60, 3249 (1991).

${ }^{20}$ T. Nakayama, in Computational Physics as a New Frontier in Condensed Matter Research, edited by H. Takayama et al. (The
Physical Society of Japan, Tokyo, 1995), p. 21.

${ }^{21}$ H. Shima and T. Nakayama, J. Phys. Soc. Jpn. 67, 2189 (1998).

${ }^{22}$ K. Slevin, J.-L. Pichard, and P.A. Mello, J. Phys. (France) 6, 529 (1996).

${ }^{23}$ M. Henneke, B. Kramer, and T. Ohtsuki, Europhys. Lett. 27, 389 (1994).

${ }^{24}$ B. Shapiro and E. Abrahams, Phys. Rev. B 24, 4889 (1981).

${ }^{25}$ D. Vollhardt and P. Wölfe, Phys. Rev. B 22, 4666 (1980).

${ }^{26}$ T. Brandes, B. Huckestein, and L. Schweitzer, Ann. Phys. (Leipzig) 5, 633 (1996).

${ }^{27}$ J.T. Chalker and G.J. Daniell, Phys. Rev. Lett. 61, 593 (1988).

${ }^{28}$ C.M. Soukoulis and E.N. Economou, Phys. Rev. Lett. 52, 565 (1984).

${ }^{29}$ H. Grussbach and M. Schreiber, Phys. Rev. B 51, 663 (1995). 